\title{
REsound the UNseen
}

\author{
Clio Flego MediaAC programme \\ Danube University Krems (AT) \\ clio.flego@gmail.com
}

\begin{abstract}
As a political phenomenon, biodata collection is not dedicated to surveillance and control regimes, neither only to medical nor health devices. A wide range of interactive installations have been made transforming organic objects into new forms of insight, addressing some ethical and political issues concerning new perspective that such sonic exploration allows in a visual-based era. Sonification practices have been used bending human reality perception to the embodiment of what is normally unseen. Can both body and sound be seen as act of resistance in the notrevolutionary world? Are them both triggers of consciousness in this paradigm, or an instrument of reassurance? What is their role in the redefinition of orality and non-linguistic forms of communication? An infinite possibility of connections and relational dynamics make both body and sound able to give shape to invisible acts of resistance, so what their in-deep position in the ecosystem governance?
\end{abstract}

body, biocontrol device, biodata environment, sonification, resilience, resistance, relational dynamics.

\section{INTRODUCTION}

By a sonification perspective, body is not only a collection of biological data, but also a complex data set build on the perception, cognition and interaction with the environment. More generally speaking, body is the core component of social structure and therefore of reciprocity and trust, being ultimately the main subject of politics of control. "In a sense, organisms have ceased to exist as objects of knowledge, giving way to biotic components, i.e., special kinds of information- processing devices". (Haraway, 2016; 35)

Technology developments play a crucial role in the common body perception, creating distance among people physically near and making feeling closer far away people. The interposition of the cybernetic communication among biotic components mutates human interventions and evolves social needs, conferring to bodies different roles and understanding of experiential and political phenomenas.

In this paradigm, sound reaffirms its power of immediacy, coming along the importance of the physical presence and the question of being visible, reflecting how appearance is shaped by disappearance, and emphasising the support ultimately required to sonic agency for emancipatory practices and political transformation.

\section{RESEARCH METHODS}

The research is focused on the strategic position of technology in relation to body and sound, contextualised in its evolution over the second part of the XIX century. The relation of the two mentioned elements has been analysed by their intersection points, which are playing a political role in the contemporary geopolitical asset, assisted by innovative sound making possibilities and different perspective on the body meaning, which in turn triggered progressive scientific experimentations and artistic researches at different levels.

Tech-artists play a crucial role in the field as both developers as well as performers, using sound as an artistic malleable material and body as harmonic instruments. If on one hand they leverage the complexity of meaning that the sonic agency implies in their usage as resistant and resilient act, on the other hand, in some cases their stance challenge the imposed biocontrol power. Body sonification is indeed a relevant tool which often has been used for medical application supporting scientific researches, but it is also an instrument for emotional control and instinctive body reactions, which has been used extensively for military operations. For each application, different intents are beyond its usage, whereas the artistic perspective generates a critical approach prompting creative uses in media arts installations and performances. Data are the essential connection point in this research, being constantly a discerning node in the inferred relations.

This research does not pretend to give an exhaustive explanation of all the existent devices leveraging sound in the context of control systems, but it is rather focused on more relevant instances related to recent media arts works using these kinds of technology scoping the sonic agencies.

Even each medium has its peculiarity, ultimate apparatuses are often hybrid combination of different technologies, existing only due to the cooperation of different systems.

Below, this production approach is particularly visible on the more recent creations, whereas all the listed devices are still on use and available on the actual market.

\section{BODY SONIFICATION WITHIN DATA ENVIRONMENTS}

Science and technology researches are commonly determined by information processing and a structured relational system, which are deeply 
involved in the IT conception of the biotic components seeing the organism in juxtaposition to the machine. As Donna Haraway wrote, "organism has been translated into problems of genetic coding and readout" (Haraway, 2016; 35), emphasising the roots of both machines and organisms in coded devices and their intimacy with power. The same question of gender changed its meaning in the postmodern world of cyborgs, roughing out a reality in which binarism lacks a real representation, yielding to a microelectronic determinism based on ubiquity and invisibility. Remarking Haraway 's theory: "Biological organisms have become biotic systems, communications devices like others." (Haraway, 2016; 60)

Since the second half of 1900s, Marcuse and Merchant analysis envisioned the integrated organic bodies in order to intensify the resistance to the predominant sovereignty. Indeed, if on one side the cyborgs would definitely fight the superimposition of the planet control, on the other side the mediated relations will directly connect the electronic components with bodies stimuli and personal identities, assimilating animals, humans and machines in an unique entangled environment. The machine of control aims to see multiple approaches simultaneously, revealing all the possible visions in once and excluding time and space limits. With enhanced bodies, the consciousness - in its direct or simulated shapes

— straightly interact digitally with 'smart environments', allowing the digital perception of organic bodies with trackable devices. The physical presence detection becomes indeed a fundamental tool in many different fields, from medicine to army and transportation systems, locating data at the base of information exchange.

New possibilities for collecting data at low and affordable costs allowed benefits democratisation, opening up new perspectives for a broader audience given also to the DIY technology dissemination. Biodata devices consequently grow as a market and much improved is given by the biomedical science affordance, meanwhile sensitive sensors enhancement refines bio-signals detection and interpretation. In the case of the EEG biofeedback for instance, it is used for controlling all those body processes that usually are involuntary. EEG training is employed for detecting epilepsy causes, and the related therapy used for patients affected by headache and migraines, high blood pressure and attention deficits. On an artistic perspective, while biofeedback was monitoring the physiological state and passively translating the signals into sonification, biocontrol introduced the idea of the systematic, reproducible volitional interactions using physiological data as input. (Tanaka, 2009) Align so with the conception of sound as immediate and sonification as a method, it defines the "data-dependent generation of sound, [in which] if the transformation is systematic, objective and reproducible, so that it can be used as scientific method." (Hermann et. al., 2008)
These systematisms often ripe in military environments linked to pattern recognition and reproducibility in the ultimate technical terms. As mechanism of biocontrol, that is the cause and the consequence of a more complex systems of power involving the body in a redefinition of its physical and political meanings.

In this context, particular relevance is attributed to the common definition and usage of the technical tools belonging in particular on the politics of controls, radically embedded in wars dynamics and data flow analysis, which link bodies and sound on their functional duality concerning both domination and resistance.

\section{BIOCONTROL DEVICES}

Bodies are identified in the spaces with physical detectors, providing significant information on movements, locations and interactions. Data coming from the physical bodies are likewise necessary, indispensable to cognise the otherwise imperceivably and instinctual human reaction to certain external stimuli. In both cases, the social relevance of these measurements is at the base of biocontrol systems, which combination produces a relevant data environment which concrete consequences are yet to tackle.

The body information is mainly detected by sensors applied on human skin, even if data mapping is often enough to create a consistent reproduction of the body identity. Signal amplification or data sonification are the principal procedures enabling the sound breeding coming from human bodies detections, and four systems are here pinpointed as main tools used for extracting biodata.

In particular, they are:

Biofeedback systems;

Radio Frequency Identification (RFID);

Biometric data system;

Machine Learning and Al.

Part of the Biofeedback systems are the biometric devices used for human recognition based on bioelectrical signals, such as Electroencephalogram (EEG), Electrocardiogram ( $\mathrm{E} \mathrm{C} \mathrm{G} \mathrm{/} \mathrm{E} \mathrm{K} \mathrm{G} \mathrm{),} \mathrm{E} \mathrm{I} \mathrm{e} \mathrm{c} \mathrm{t}$ r o $\mathrm{m}$ y o g r a m ( $\mathrm{EMG}$ ), Mechanomyogram (MMG), Electrooculography (EOG), Galvanic Skin Response (GSR) and BioMuse systems.

In the case of Biometric data system, based on body measurements and calculations processed by computer analysis, it is here distinguished the voice of 'Biometrical control application' in the subchapter 4.3.1 due to the relevance in the field and the technical specificities. This part is indeed focalised only on applications made for mobile devices, consecrating the new technological era based on mobility and small smart systems. 
Different provenience and purposes produced these kinds of devices over the last decades, with the main intent to control the human body in its intimacy. Some of these apparatuses will be here below take in consideration singularly, analysing their interposition in between human bodies and sound in their artistic expressions, with the intent to give voice to human physical presence in a mute Anthropocene in which the revolution is speckless and the expression of the self-distorted.

\subsection{Biofeedback system}

Biofeedback systems were invented in order to gain awareness on some physiological activities connected with electrical impulses, which use to be imperceivable to normal human senses and with the intent to manipulate their functions.

Biofeedback was used as an instrument for giving voice to the inner part of the person: ancient Hindu were apparently using this technique as exercise control over breathing and physiological processes, meanwhile in the 1960s Western countries spiritual tendency push an intense experimentation with extrasensory perceptions using computational power for expanding human sensation. On the other side the importance of collecting biodata grown in order to expand human abilities and as a tool interlacing physiological properties of the performers' bodies with the computational quality of electronic instruments. In 1964, the American pioneer composer Alvin Lucier was using his own sonified brain waves in order to compose Music for solo performer (Fig. 1). Other artists in the same period, such as John Cage and David Tudor, as well as Barbara Mayfield, Richard Teitelbaum and Frederic Rzewski, worked on the translation of signals into sounds, until arriving in the 1970s in which David Rosenboom connected the brain signals to an analog modular synthesiser. It was only few years before the more florescent activity of live electronic music. In this particular field, the main technique used were EEG, EMG and MMG, with which artists we re creat ing i m provis a t i o $\mathrm{n}$ a $\mathrm{n} \mathrm{d} \mathrm{m}$ u s i c performance in real time.



Figure 1: Scheme for 'Music for Solo Performance' drawn by Alvin Lucier. Image courtesy: MusikTexte, 2005. In 'From Invisible to Visible, the EEG as a tool for Music Creation and Control' by Alberto Novello.
Medical apparatus applications in bio-signal-driven art switched their enrolment from devices employed for diagnosing pathologies to emotional interaction instruments in performances, embracing the challenge of making the viewers meaningful interlocutors in the artwork. The spectators became co-active in the performances, conveying their bodies to the shared space and pushing their limits of physiological understanding. The bodies significance were indeed having a metamorphosis, and new related devices slowly started to appear on the scene. BioMuse system

The BioMuse system was founded by Benjamin Knapp and Hugh Lusted in 1988, after a continuative work on a number of neurotechnology projects. It was developed as an eight-channel bio controller, able to read EEG, EMG, EOG and EKG data. During the 90s, it was purchased by numerous R\&D facilities and used in universities, corporates and military lab, among others, NASA, US Air Force and Honeywell. It was employed for capturing and processing a variety of bioelectric signals and as neurophysiological interface, controlling the humans involuntary reactions. (Cavuoto, 2011) The system has also been used as video games controller device after being utilised for monitoring physiological variables in medicine and psychophysiology, improving the human- computer interactions and their correlations to emotions. The relevant contribution at SARC (Sonic Art Research Centre, Belfast) facilitated the technical aspect, while the social and artistic applications gave an essential contribution in the field.

Its inventor Ben Knapp, Digital Luthier creating instruments and active from the 1990s in CyberArts, since 30 years is working on new ways to produce sounds and reveal the invisible, considering what is inside human bodies as an essential source of music and emotions. In collaboration with Eric Lyon and Gascia Ouzounian, Knapp founded the ensemble BioMuse Trio, composing music from physiological signals modulated by measured performers' bodies movements. The music evolves accordingly to physiological and emotional variations, which in some compositions such as Stem Cells are also combines with audience's skin and heart rates signals recorded with GSR (Galvanic Skin Response) installed on some viewers during the act. In the similar composition Biospaces, what interested the artist, even more than the physiological indicators tracking emotion to create music, was the production of a feedback loop allowing the performers to jam with the audience sound response. In this case, the interrelation of the bodies was showing a unique effect in which differences were not audible anymore, disrupting eventual system of power created by the different roles people in the room were having. The machine interposed its agency in between bodies, revoking any kind of contrast among participants. 4.1.2 Galvanic Skin Response (GSR) 
This mix of composition and improvisation is an essential part of BioMuse Trio's works, but also other media artists used this technology for

experimentation with sound.

For instance, Claudia Robles-Angel in the GSRbased installation Skin (2012-14) used the electronic signals during the performance in order to measure the state of visitor's excitement. The GSR interface indeed sizes the skin's visitor moisture as a reflection of the neurological and emotional states.

Contextually, the artist focused on the space of the unexpected connected with the mangling bodies, not specifically linked with measurements in itself, but rather with the instinctual subjective emotions expressed regardless the machine control. Sound suddenly acts as a resilient material in the invisible spectrum, such as malleable volume able to adapt to imposed systems and to open up to plural possibilities.

This system in particular has been used for biopolitical marketing such as recording emotional appraisal, subconscious decision processes during account purchases or in order to identify target audience and personas.

Exactly in this paradigm is when the voices lose their frequencies, the words suddenly became empty, and the bodies enact with a spontaneous act of resilience. The act of listening becomes a space of intimacy and participation that needs time and space to emerge and gives to language an incapacity of expression. The body therefore unveils something otherwise not perceivable through the technological and social subjective mediations, becoming a real act of resistance in a liminal space. (LaBelle, 2018)

\subsection{Radio Frequency Identification (RFID)}

Radio-frequency identification is a technology used in many industries, such as the pharmaceutical or automobilist, and is also at the base of the microchip implanted in animals for positive identification.

Recently Disney announced the relying on the market of bracelets with RFID tags for tracking children. More specifically, it is based on the use of electromagnetic fields to identify and track tags attached to objects, which is readable by passive or active tags that activate by proximity interrogating radio waves.

The ancestor of this kind of instrument is the listening device invented by Léon Theremin in 1945. Even though Theremin device was not an identification tag, it was passively energised and activated by outsource waves. Originally produced for the Soviet government as a proximity sensor to be employed during the Russian Revolution, Theremin finds its success during the two world wars, being then world wild recognised as an avant-garde electronic instrument. It is based on two metal antennas perceiving physical movements without contact, meanwhile the performer controls frequency and amplitude with hands movements in order to produce music with the amplified electronic signals.

This reflection on sounds creates a different interpretation, listening and perspective of the body, whereas experimental sound practices act as a medium embarking historical endeavours of activism, preservation, gender, citizenship, empowerment, resistance and insubordination. The sound was unwittingly reflecting the counterpart of the official versions exploring new ways of experiment consciousness, giving at the same time amplification to the unheard voices, victims of the contemporary wars, as well as to the people opposed to the regime: while the hands movements were sinuously orchestrating the invisible, the ghostly sound were raising secret feeling and concealed emotions.

\subsection{Biometric data systems}

The technological mediation has become in the worst cases a mere interface, meanwhile ubiquitous biocontrol systems are used for identifying physical or behavioral characteristics. Based at list on one type of sensor, fingerprints readers, DNA analysis, signature dynamics, voice and facial patter recognitions are only few technologies using person information for human classification, social security and targeted marketing.

Exemplarily explained by Yoon Chung Han in Eyes (2018, Fig. 2), the importance of persons' own identity is showed in the participative installation by the contingent disembodiment of identities through their digital representation. The biodata taken by the visitors are indeed used actively in the installation, based on humans' iris data collection and consequent transformation into harmonic sounds and $3 \mathrm{D}$ animated sculptures. By the artist's statement, digital era main problems using the biometric data are the privacy violations and the criminal use of personal data, which identity can be stolen or misused. The artist gives to participants the chance to explore their personal identities through the comparison with others iris-based identities, taking over the problem of 'disembodied' identities by subjects' personal experience. In this way, their individual (audiovisual) representation improves the perception of the self, mirroring the actual condition by the simulation of the real.

Remarkable the considerations on bodies identities in the digital era addressed in the book Testo Junkie: Sex, Drugs, and Biopolitics in the Pharmacopornographic Era by Paul B. Preciado. He defines the body as cultural and political archive, place able to unconsciously manifests identities and unequivocally represents the inner self instinctively, at the same time in which the surrounding rely on new control camera and recognition systems. (Preciado, 2013) 


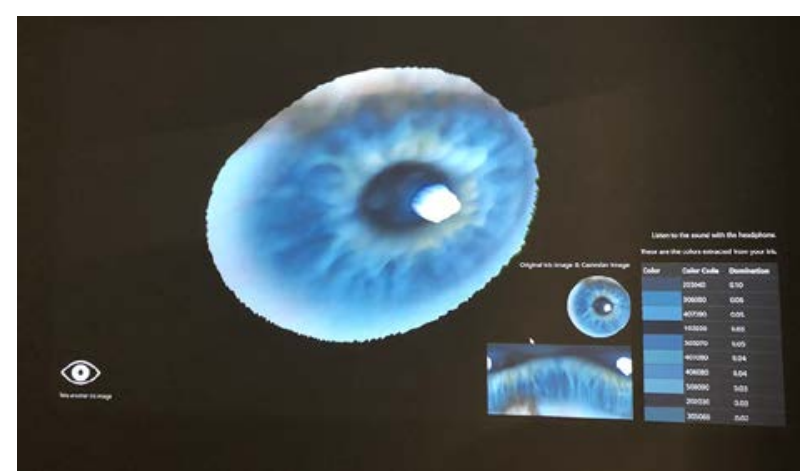

Figure 2: Visitors' Iris digitalisation process, for the installation 'Eyes' (2018) by Yoon Chung Han. Retrieved from yoonchunghan.com/portfolio/Eyes.html

Based on biometric data, but this time given by face recognition, is the interesting approach of Ryo Ikeshiro in Ethic Diversity in Sites of Cultural Activity (2015). Posing the question if a computer can be racist, sounds and images produced by the machine give different results depending on the visitors' ethnic diversity and where the exhibition is displayed: for example in Vienna, Johann Strauss's The Blue Danube is selected for light skins, meanwhile Fela Kuti's Zombie is the one that was morphed for dark skins. This shaped racial profiling drawn the attention to the technological agency, that even is seemingly a neutral entity, it actually reinforces the established structures of power.

In this scenario the re-appropriation of the space through the sound remarks the human necessity of expression and expansion, fighting against the body annihilation consequent to the economical oppression and socio-political control executed by the machine. In an era in which the visual perception of the body is completely distorted by the technological mediation and its representation, the data sonification obtains the political position of identity material reappropriation, giving immediacy to unconscious thinking otherwise remained unexpressed.

\subsection{Biological control applications}

As DNA chain and protein molecule store massive amounts of biological information in nano millimetre particles embedded in bodies, the innovation of computational system is aiming to create quantum processors in order to acquire infinite quantity of data. This process involves scientific and technological researches in public and private domains, often leveraging social networks and accessible databases for increase private enterprises incomes.

This memory segmentation due to digital organisation is reflected at micro and macro levels, sometimes involving personal data identity fragmentations, some other times engaging network information spaces. At the intersection of these concepts, are fascinating the DNA Indexes, created for carrying millions of genetic tests by the Federal Bureau of Investigation in USA. In order to classify criminals, identify soldiers, test new weapons, decide paternity cases and so on, the Indexes make available thousands of data collected legally and illegally by different sources. The fear of these databases is the inappropriate use of information by unauthorised cases, such as stigmatising genetic illness or identifying persons, taking important decision based on patients private medical and legal histories.

Artists such as Kyle Evans or Markus Buehler reflect on these socio-political issues, using both nanotechnology information extraction systems and Al for representing the complex structures of DNA and protein through their sonification. Kyle Evans explains how easier is memorise and recognise musical tones instead of alphanumeric string, and that the DNA can plays beyond the compositional control of the composer resulting less versatile of a representation, giving more certain idea of truthiness. (Evans, 2009)

Agree with Evans' idea about sonification as an instrument for gaining knowledge via listening experience, Markus Buehler at the MIT lab translates proteins into music, acquiring insight into the structure and creating new possible chain variations. Tones and scales are based on each amino acid molecule, computed using quantum chemistry theories. For now, 20-tone scales have translated amino acid sequences creating blocks of proteins, but Buehler and his team wish to glean new insights, also thanks to the support of the Al system. It catalogues melodies and introducing slight changes in the music sequence the system is able to create completely new sequences, then translated from the top into sounds with the ability to create a new biological scenario.



Figure 3: Screenshot of 'Amino Acid Synthesizer' application to download on Google Play Store.

The researchers use data not only for the mentioned sonification, but also in the free application for smartphone 'Amino Acid Synthesizer' (Fig. 3), which plays the sounds of amino acid and record protein sequences as musical compositions.

Marketing expanding in this era is entangling the biopolitical framework in their strategies, using always more often ubiquitous technology in order to identify 
users' social behaviours, cognitive capacities and bodily conduct. As Nike+ mobile application tracking users' training and capacities in order to exploit the collected data for target their marketing dimension and push forward consumers engagement (Charitsis et. al, 2018), many wearable technologies as smart watches and mobile applications are employing their efforts in enhancing this data-capitalised paradigm. Strava's application is another relevant example: if, on the one side, a fitness tracking app is used for improving cyclists performance and runners timing; then, on the other side, making its data public it pinpointed secret US army bases in Afghanistan, Djibouti and Syria, giving away sensitive information about the militants using locally the application. (Hern, 2018; Fig. 4)

As the subcomandante Marcos wrote from the mountains of Chiapas, with not particular regard to what the XXI century algorithms does in contemporary people's reality view, neoliberalism "implies the destruction of humanity as a sociocultural collective and reconstructs it as a marketplace". It was a strong anticipation of how new capitalism absorbs new initiatives created outside its system in order to contrast it totalitarianism and embed them in an exploitative paradigm. In the contemporary daily life, appearance of collectivity is in its reaffirmation over the subjectivity.

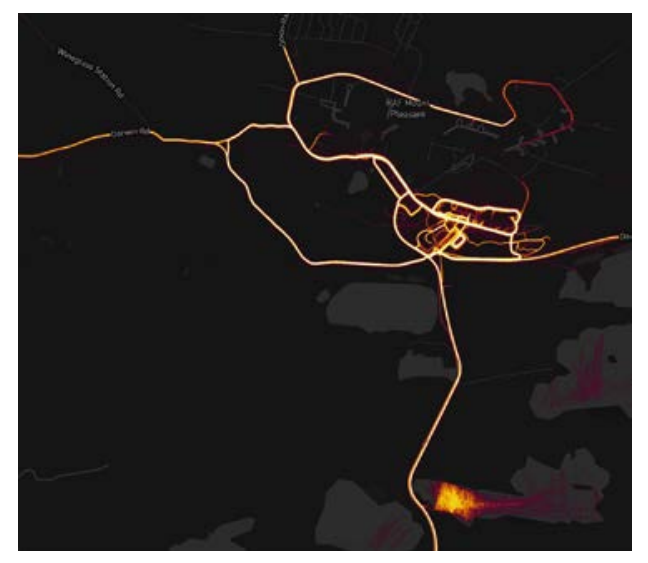

Figure 4: RAF Mount Pleasant, Falkland Islands. Image: Strava heatmap. Retrieved from The Guardian's online article: Sept 28th, 2018.

Machine Learning and Artificial Intelligence Machine Learning (ML) and Artificial Intelligence (AI) are actually used for digital medical consultation, precision surgery and ultimately for skin cancer detection. Also, they have been extensively employed for military actions, such as the Lethal autonomous weapons (LAWs), robot able to independently search and engage target in battlefield, or the MQ-9 Reaper, lethal military drone piloted via satellite, driven by artificial intelligence and based on human sensors operation for drops the bombs - or fires - on the chosen target. In some devices, it is also implementing face recognition systems, as Google's Project Maven incorporating TensorFlow Al in US military drone for analysing footage in order to detect objects and unclassified data.

Notable in this section is Evelyne Drouin's interactive installation Al see you (2018), which uses Al for seeing and hearing viewer facial

features and gestures in order to analysis emotional states and listen to musical patterns and rhythms, creating a unique sonic environment as a creative machine creation. The Al system indeed turns the acquired data in instrument generating the soundscape and animating a giant illuminated sculpture, with the intention to reflect on human vision on the machine and human perception of the machine vision, trying to understand consequent misjudgements and biases. Assumed that data are feeding Al algorithms with the purpose to manipulate the masses, Oliver Rack asked in the conference smARTplaces | INNOVATION in Culture, taking place at ZKM Karlsruhe as part of the EU project 'smARTplaces' in March 2018: "Do we want our citizens surrender their private data to companies and leave them alone with this? (..) Do we want to feed foreign economies with added values by exploiting these data? And selling Artificial Intelligence as a service we don't have our own?". (Rach, 2018) The $\mathrm{ML}$ and Al market is one of the more discussed nowadays, on both ethic and social levels. The intelligence created artificially by data accumulation is essentially based on machine learning applications, fed by human personal willingness to share their own data in public domains for free. At the cornerstone of this dynamic, the importance of the body presence became once again a relevant field to discuss, in which sensing goes beyond the physical body limitations. Already in the 1960s McLuhan wrote in his electronic age analysis that electronic unfold the body and consciousness toward a generalised externalisation of feelings, knowing and sharing. (McLuhan, 1964)

According to his writings, the extension of the nervous system provoked by the constant presence of electronic devices (that for what concern Franco Berardi it rather arrives to the soul) produces a certain level of vibration intertwining the processes of network culture, the one yielding more properly the identity definition through movements in the social coexistence. This reflects on the position of the bodies in a datafied space, in which the total interconnection gives primary importance to the visual as much as it is a simplified reflection of that complexity exclusively expressed properly by words.

\section{CONCLUSION}

Sound intervention is an essential element of many visual artworks as a symptom of an emerging requirement of social re-appropriation. In an era based on constant recording with digital control systems, the usage of data coming from an involuntary flow is essential for understanding the complexity of the sound production in this context. 
Human bodies become unconscious source of data for the sound compositions. No prepared movements nor any direct action of the artists are involved during the listening moments, but only the spontaneous interactions of the present bodies in all their declinations and variations with the devices through the data flow detection.

The body annihilation subdued to the growing presence of the technology (in its role of mediator) reacts in these processes as a resilient material which expands its power mainly through its digital representation, showing the process of making instead of a proper final artistic work.

Many devices built for biocontrol finalities become simultaneously instrument for the political machine and components for amplifying the more inner instincts and human emotions, letting emerge the instinctual reactions. Artists are in this paradigm upholders and opponents as one, invoking the presence of the sonification as medium and tool for criticality.

\section{As Judith Butler wrote:}

Where sound, body and space meet, new dimensions of, and sensitivities towards, environments can be engaged, and our relationship to these and to ourselves and each other within these can be re-imagined and transformed. (Butler, 1988; 521)

That is exactly the moment in which sonification become important: when the intention of the artist is only making visible uncontrollable processes, avoiding the same repetitive physical gesture needed in order to produce music. This overlapping of the processes and the final artworks is what enable embodied imagination and open new ways to face and interpret reality, giving the chance to the bodies to finally express their inner movements without control and external political concerning.

\section{REFERENCES}

Butler, J. (1988). Performative acts and gender constitution: An essay in phenomenology and feminist theory. Theatre Journal, 40, 519-530.

Cavuoto, J. (2001) 'Biocontrol Corp. Looks to Break Into Computer Peripheral Market', in Neurotech b u s in es s report. Re trieved fro m www .neurotechreports.com/pages/ biocontrol.html (Last access: 30 August 2019)

Charitsis, V., Yngfalk, A. F., \& Skålén, P. (2018). 'Made to run': Biopolitical marketing and the making of the self-quantified runner. Marketing Theory. doi.org/10.1177/1470593118799794

Evans, K. (2009) Artist as Scientist: The Sonification of DNA. Retrieved from

https://www.academia.edu/11659690/

Artist_as_Scientist_The_Sonification_of_DNA

Haraway, D. (2016). Manifestly Haraway, University of Minnesota Press, 2016. ProQuest Ebook Central, http://ebookcentral.proquest.com/lib/

warw/detail.action?doclD=4392065.

Hermann, T. (2008). 'Taxonomy and definitions for sonification and auditory display', in ICAD2008, Proceeding of the 14th International conference on Auditory Display. Paris, France: IRCAM.

Hern, A. (2018). 'Fitness tracking app Strava gives away location of secret US army bases' in The Guardian. Retrieved from www.theguardian.com/ world/2018/jan/28/fitness-tracking-app-gives- awaylocation-of-secret-us-army-bases (Last access: 30 August 2019)

LaBelle, B. (2018). Sonic agency: Sound and emergent forms of resistance. London: Goldsmiths Press.

McLuhan, M. (1964). Understanding media: The extensions of man. New York: McGraw-Hill.

Preciado, P. B. (2013). Testo Junkie: Sex, Drugs, and Biopolitics in the Pharmacopornographic Era. CUNY: The Feminist Press.

Rach, O. (2018) Audience Insights and Open Data. smARTplaces | INNOVATION in Culture, ZKM Karlsruhe, March 2018. Retrieved from www.youtube.com/watch?v=RO1tdLOzN8g.

Robles-Angel, C. (2011). 'Creating Interactive Multimedia Works with Bio-data', in NIME2011 International Conference on New Interfaces for Musical Expression. Oslo: NIME2011. 421- 424.

Robles-Angel, C., Scherffig, L., Birringer, J., Seifert, U., Schmidt, L.. (2017) 'Bio-medical Signals in Media Art', in ISEA2017 Proceedings. Mazinales Biocreation and peace. Bogotá: ISEA2017. 720- 29.

Tanaka A, (2009). 'Sensor based Musical Instruments and Interactive Music', in Dean, R. (eds), The Oxford Handbook of Computer Music. Oxford, UK: OUP. 233- 257.

Teitelbaum, R. (1966-74). 'Tune: Some Early Experiments in Biofeedback Music', in Rosenboom, D. (eds) Biofeedback and the Arts, Results of Early Experiments. Aesthetic Research Centre of Canada Publications, Vancouver. 1976, 35- 56.

Vesna V. (2007), Database Aesthetics. Art in the Age of Information Overflow. Minneapolis, London: University of Minnesota Press. 